\title{
Entwicklung eines Augmented Reality Assistenzsystems zur Unterstützung von Instandhaltungsaufgaben in der Produktion
}

\author{
Ruben Schlagowski ${ }^{1}$, Claudia Meitinger ${ }^{2}$, Lukas Merkel ${ }^{3}$ \\ ${ }^{1}$ ruben.schlagowski@hs-augsburg.de \\ 2 claudia.meitinger@hs-augsburg.de \\ 3 lukas.merkel@igcv.fraunhofer.de \\ 1,2 Hochschule für angewandte Wissenschaften Augsburg, Deutschland \\ ${ }^{3}$ Fraunhofer Einrichtung für Gießerei-, Composite- und Verarbeitungstechnik IGCV, \\ Deutschland
}

DOI: 10.14464/awic.v3i0.250

\begin{abstract}
In vernetzten Produktionsstätten werden die Tätigkeiten der Mitarbeiter vielfältiger und komplexer. Zudem müssen die wenigen verfügbaren Mitarbeiter flexibel eingesetzt werden können. Assistenzsysteme können dazu beitragen, die Qualifikation der Mitarbeiter an die steigenden Anforderungen anzupassen.

Dieser Beitrag befasst sich mit der Entwicklung eines Assistenzsystems für die Unterstützung von Maschinenbedienern bei der Durchführung von Instandhaltungsaufgaben in der Produktion. Ein Prototyp des entwickelten Systems benutzt regelbasierte Algorithmen und ein Augmented Reality Headset um bei der Inbetriebnahme und Entstörung eines MechatronikTrainingssystems zu assistieren.
\end{abstract}

Keywords: Assistenzsystem, Augmented Reality, Produktion, Instandhaltung

\section{EINFÜHRUNG}

Mit dem Wandel hin zu einem neuen Industriezeitalter ändern sich die Anforderungen an Mitarbeiter maßgeblich. Diese werden in den zukünftigen „Smart Factories“ zunehmend zum flexiblen Bindeglied, welches spontan und vielseitig im Produktionsprozess eingesetzt werden kann. Diese geforderte Flexibilität erhöht die Anforderungen an die Qualifikation für die Mitarbeiter signifikant, besonders aufgrund der benötigten hochfrequenten Wissens- 
aktualisierung "on demand“ und der Ausweitung der Kompetenzen auf ein breiteres Aufgabenumfeld (Mülder 2016, S. 22-33). Vom Wandel betroffene Tätigkeitsumfelder sind auch die Maschinenüberwachung und Entstörung in der Produktion. Assistenzsysteme können hier Mitarbeiter entlasten und unterstützen, sodass es für sie möglich wird, den steigenden Anforderungen nachzukommen und neue Fertigkeiten zu erlernen.

Augmented Reality wurde in diesem Zusammenhang bereits in mehreren Nutzerstudien als vielversprechende Technologie für die Mitarbeiterassistenz im industriellen Umfeld bestätigt (Friedrich 2004) . Dennoch schafften es viele der entwickelten Assistenzsystem nicht über den Prototypenstatus heraus. Als ein Grund für diesen Umstand wird die fehlende Intelligenz der Assistenzsysteme genannt (Junghans et al. 2014, S. 331). Ein Ansatz zur Lösung dieses Problems ist die Zuschaltung von Experten, welche den Laien mit Hilfe von Augmented Reality Instruktionen vermitteln (Masoni et al. 2017; Wang et al. 2014). Weitere Ansätze basieren auf der automatisierten Auswertung von Videomaterial mit Hilfe von Machine Learning (Petersen und Stricker 2015) und der Modellierung von Situationsbegebenheiten mit Hilfe von Graphen und Ontologie-basierten Sprachen (Wang et al. 2016; Zhu et al. 2014).

Das in diesem Beitrag vorgestellte Assistenzsystem begegnet den genannten Herausforderungen mit Hilfe einer regelbasierten kognitiven Architektur, welche eine flexible Erfassung und Interpretation der Situation ermöglicht. Auf Grundlage dieses Situationsbewusstseins kann das System Aktionen zur Problembehebung planen und durchführen.

Um das für eine Situationsinterpretation notwendige Wissen über das Arbeitssystem abzubilden, wird der Nutzungskontext des Systems analysiert und mit Hilfe von Zuständen und Zustandsübergängen beschrieben (siehe Kapitel 2). Der im Anschluss beschriebene Prototyp des Systems kann auf Basis dieses erhobenen Wissens eine Möglichkeit der Fehlerbehebung auswählen. Dies kann die eigenständige Bedienung der Maschine oder die Anleitung eines Mitarbeiters bei der Fehlerbehebung sein.

\section{ANALYSE DES NUTZUNGSKONTEXTS}

Ein zentraler Bestandteil des menschzentrierten Entwicklungsprozesses zu Entwicklung von gebrauchstauglichen interaktiven Systemen ist die Analyse des Nutzungskontexts (DIN EN ISO 9241-210, S. 17-18) inklusive der Arbeitsaufgabe. Diese wird als die „zur Erreichung eines vorgesehenen Arbeitsergebnisses erforderliche Aktivität oder Anzahl von Aktivitäten des Arbeitenden“ (DIN EN ISO 6385: 2016, S. 9) definiert.

Im untersuchten Anwendungsfall der Instandhaltung einer Produktionsanlage ist das angestrebte Arbeitsergebnis die Herbeiführung und Beibehaltung eines Zustands der Produktionsanlage, in dem das gewünschte Produkt fehlerfrei produziert wird. Die erforderlichen Aktivitäten des Nutzers, die zum Erreichen bzw. Aufrechterhalten dieses Zielzustands nötig sind, wurden u.a. durch Selbstbeobachtung analysiert und in wiederkehrende Sinnabschnitte (Teilaufgaben) gruppiert (Schlagowski et al. 2017). Das 
erhobene Wissen wurde dabei so strukturiert, dass wie bei der Ziele-Mittel-Analyse (meansends analysis, (Newell und Simon 1972)) nach der Durchführung einer oder mehrerer Teilaufgaben jeweils ein eindeutig nachvollziehbarer Zustand der Produktionsanlage vorherrscht, ein sog. Teilziel (vgl. Abbildung 1: Kreise mit „TZ“).

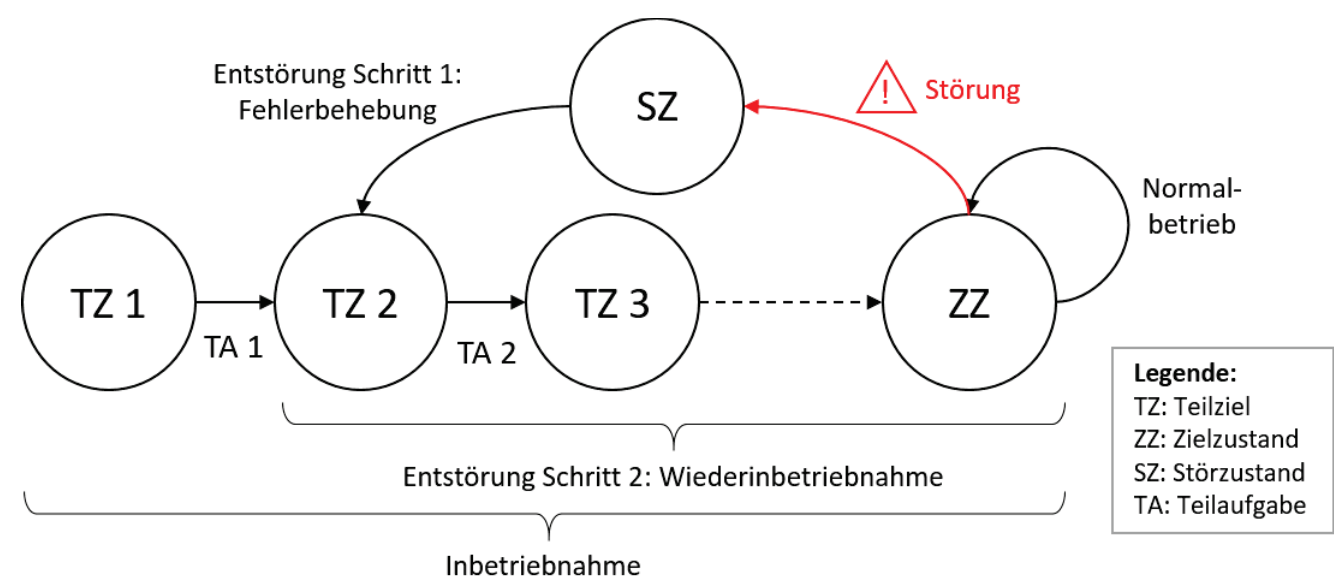

Abbildung 1: Entstörung und Inbetriebnahme als Verkettung von Teilaufgaben und Zuständen Quelle: $\quad$ eigene Darstellung

Die Anzahl an erforderlichen Teilzielen kann variieren und ist von der Abweichung zwischen dem aktuellen Zustand und dem Zielzustand abhängig. Die größtmögliche Abweichung im aktuellen Anwendungsfall befindet sich zwischen einer komplett abgeschalteten Produktionsanlage und dem anvisierten Zielzustand einer aktiven und fehlerfreien Produktion. Um diese Differenz auszugleichen, muss die Arbeitsaufgabe „Vollständige Inbetriebnahme der Produktionsanlage" bearbeitet werden. Tritt eine Störung während der Produktion auf, wird ein Störzustand (vgl. Abbildung 1: SZ) erreicht, der durch die Behebung des aufgetretenen Fehlers zu dem Zustand eines Teilziels zurückgeführt werden kann. Anschließend muss der Zielzustand durch eine Wiederinbetriebnahme wiederhergestellt werden.

Um die gewonnen Erkenntnisse über die Arbeitsaufgabe in ein Assistenzsystem implementieren zu können, müssen alle Teilziele, Störzustände und Teilaufgaben analysiert und beschrieben werden. Tabellen 1 und 2 zeigen einen Auszug für die Analysen der Teilziele und Störungen für den beispielhaften Anwendungsfall der Bedienung und Instandhaltung eines Mechatronik-Trainingssystems in der Lernfabrik des Fraunhofer IGCV in Augsburg (siehe Abbildung 2). Dieses System (von nun an Produktionsanlage genannt) entnimmt Würfelhälften aus verschiedenen Magazinen, verpresst diese mit Pins und lagert das Endprodukt in ein Hochregallager ein. 
Tabelle 1: $\quad$ Teilziele (Auszug) und verknüpfte Teilaufgaben im Anwendungsfall Quelle: $\quad$ Eigene Darstellung

\begin{tabular}{|l|l|l|}
\hline Teilziel & Teilaufgaben & Merkmale des Teilziels \\
\hline TZ1: Steuerungen online & 3x Hauptschalter betätigen, warten & Steuerungen antworten über TCP \\
\hline TZ2: Druckluft liegt an & Kompressor einschalten, warten & Sensorik an den Druckrelais „true“ \\
\hline TZ3: Anlage zurückgesetzt & $\begin{array}{l}\text { 3x Schlüssel drehen, 3x Taste } \\
\text { betätigen }\end{array}$ & Auslesen von Daten der Steuerung \\
\hline TZ4: Magazine nachgefüllt & 2x Lager leeren, 4x Magazin füllen & $\begin{array}{l}\text { Magazinsensoren alle „true“, } \\
\text { Bestätigung durch Operateur }\end{array}$ \\
\hline
\end{tabular}

Tabelle 2: $\quad$ Störungen (Auszug) im Anwendungsfall

Quelle: $\quad$ Eigene Darstellung

\begin{tabular}{|l|l|l|l|l|}
\hline Störung & Fehlerbild & $\begin{array}{l}\text { Merkmale des } \\
\text { Störzustands }\end{array}$ & Fehlerbehebung & Folgezustand \\
\hline $\begin{array}{l}\text { Druckluft liegt } \\
\text { nicht an }\end{array}$ & $\begin{array}{l}\text { Würfelhälften werden } \\
\text { nicht ausgeworfen }\end{array}$ & $\begin{array}{l}\text { Sensorik an einem der } \\
\text { Druckrelais auf „false“ }\end{array}$ & $\begin{array}{l}\text { Neustart } \\
\text { Druckluftkompressor }\end{array}$ & TZ2 \\
\hline $\begin{array}{l}\text { Leerung des } \\
\text { Lagers nicht } \\
\text { registriert }\end{array}$ & $\begin{array}{l}\text { Lager leer, Produktion } \\
\text { stoppt vor Station 3 }\end{array}$ & $\begin{array}{l}\text { Lagerkapazität auf } \\
\text { Maximalwert }\end{array}$ & $\begin{array}{l}\text { Zurücksetzen der } \\
\text { Lagerkapazität durch } \\
\text { Zugriff auf Steuerung }\end{array}$ & TZ3 \\
\hline
\end{tabular}

\section{SYSTEMENTWURF UND PROTOTYP}

Das Assistenzsystem das den Operateur bei der Inbetriebnahme und Entstörung des Mechatronik-Trainingssystems unterstützen soll, orientiert sich an typischen Schritten der menschlichen Informationsverarbeitung im kognitiven Prozess (Onken und Schulte 2010; Putzer und Onken 2003):

- Umgebungswahrnehmung,

- Situationsinterpretation,

- Zielbestimmung,

- Aktionsplanung und

- Aktionsdurchführung.

Diese Schritte finden sich unmittelbar in der Systemarchitektur wieder. Für die Implementierung der Architekturkomponenten Situationsinterpretation, Zielbestimmung und Aktionsplanung wird ein Kernel der kognitiven Architektur Soar (Laird 2012) eingesetzt. Dieser erfasst zyklisch die verfügbaren Informationen über den Zustand der Produktionsanlage und vergleicht diese Daten mit den bekannten Zuständen, die zuvor durch die Analyse des Nutzungskontextes erfasst wurden. Anschließend wird ermittelt, welches nächste Teilziel angestrebt werden soll und welche Aktionen dafür notwendig sind. Nachdem die nächste durchzuführende Aktion ausgewählt wurde, kann deren Umsetzung an die aktuelle Situation angepasst werden, um den Zustand des Arbeiters zu berücksichtigen. Dazu zählen die Erwägung einer direkten und eigenständigen Einflussnahme auf die Produktionsanlage, oder 
eine Anweisungsübermittlung bzw. Unterstützung des Maschinenbedieners bei der Durchführung einer erforderlichen Aktivität.

Der Prototyp nutzt das Augmented Reality Head-Mounted Display HoloLens des Herstellers Microsoft für die Nutzerinteraktion. Dieses kann dafür auf ein breites Spektrum an Ein- und Ausgabemodalitäten zugreifen (z.B. Gesten- und Sprachsteuerung, Audioausgabe und ARDisplays) und empfängt die vom Soar-Kernel generierten Instruktionen über TCP/IP. Für die Zustandsüberwachung und die direkte Einflussnahme auf die Produktionsanlage wurde ein Motion Logic Programming Interface (MLPI) des Herstellers Bosch Rexroth eingesetzt, welches Lese-und Schreibvorgänge an Zustandsvariablen der SPS-Einheiten ermöglicht.

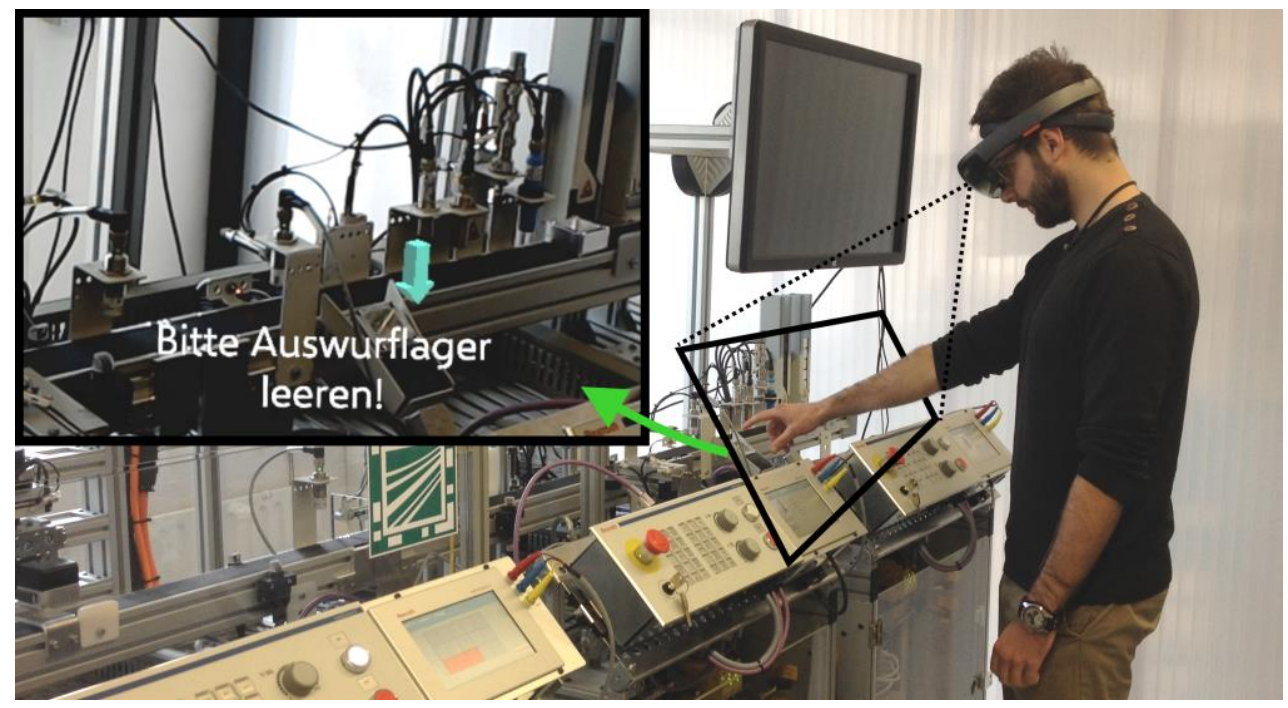

Abbildung 2: $\quad$ Sicht eines Maschinenbedieners bei der Nutzung des Demonstrators in der Lernfabrik Quelle: $\quad$ eigene Darstellung

Abbildung 2 zeigt den Prototypen im Einsatz am Mechatronik-Trainingssystem in der Lernfabrik mit der augmentierten Sicht des Maschinenbedieners. Diese enthält die Anweisung, ein durch einen Pfeil gekennzeichnetes Auswurflager zu entleeren um die Anlage zu entstören.

Um eine zeiteffiziente Entwicklung des Assistenzsystems zu gewährleisten, wurde zusätzlich ein HoloLens-Emulator eingesetzt und eine Simulations-Software entwickelt, die das Verhalten der Produktionsanlage ähnlich einem digitalen Zwilling mimen kann.

\section{ZUSAMMENFASSUNG UND AUSBLICK}

Zusammenfassend konnten wichtige Ergebnisse für die Entwicklung eines kognitiven Augmented Reality Assistenzsystems erzielt werden. So konnte mit Hilfe der verwendeten Methoden für die Analyse des Nutzungskontextes ein Teil des Verhaltens der Produktionsanlage beschrieben und simuliert werden. Weiterhin wurden die für die Kommunikation mit dem Assistenzsystem erforderlichen technischen Schnittstellen zum AR- 
Headset und zu den SPS-Einheiten implementiert und ein Testbed für die Integration der kognitiven Architektur geschaffen. Mit Hilfe dieser Software-Komponenten konnten erste Funktionalitäten auf Basis eines Soar-Kernels umgesetzt werden.

Zukünftig soll eine Anbindung von Smart Watches, welche zur Mitteilungen von Entstörungsaufträgen vorgesehen sind, implementiert werden. Weiterhin soll eine Schnittstelle für das Einpflegen von Authoring-Inhalten mit Hilfe des AR-Headsets eine benutzerfreundliche Integration von Daten über den Nutzungskontext ermöglichen. Außerdem soll die Aktionsplanung des Assistenzsystems erweitert werden, um die Expertise und die mentale Beanspruchung des Operateurs bei der Aktionsplanung zu berücksichtigen.

\section{LITERATURVERZEICHNIS}

DIN EN ISO 9241-210 (2011): Prozess zur Gestaltung gebrauchstauglicher interaktiver Systeme.

DIN EN ISO 6385: 2016 (2016): Grundsätze der Ergonomie für die Gestaltung von Arbeitssystemen.

Friedrich, W. (Hrsg.). (2004): ARVIKA. Augmented Reality für Entwicklung, Produktion und Service. Erlangen: Publicis Corp. Publ.

Junghans, A., Wodrich, K., Jeretin-Kopf, M. \& Haas, R. (2014): Unterstützung von Wartungsarbeiten durch verteilte AR-Assistenzsysteme. Technische Unterstützungssysteme, die die Menschen wirklich wollen, 328-338.

Laird, J. (2012): The Soar cognitive architecture. Cambridge, Mass: MIT Press.

Masoni, R., Ferrise, F., Bordegoni, M., Gattullo, M., Uva, A. E., Fiorentino, M., Carrabba, E. \& Di Donato, M. (2017): Supporting Remote Maintenance in Industry 4.0 through Augmented Reality. Procedia Manufacturing 11, 1296-1302.

Mülder, W. (2016): Arbeit 4.0. Smarte Maschinen für Smart Workers? Wissensmanagement (4), 22-23.

Newell, A. \& Simon, H. A. (1972): Human problem solving (1. print). Englewood Cliffs NJ: Prentice-Hall.

Onken, R. \& Schulte, A. (2010): System-Ergonomic Design of Cognitive Automation. DualMode Cognitive Design of Vehicle Guidance and Control Work Systems (Studies in Computational Intelligence, Bd. 235). Berlin, Heidelberg: Springer-Verlag Berlin Heidelberg.

Petersen, N. \& Stricker, D. (2015): Cognitive Augmented Reality. Computers \& Graphics 53, 82-91.

Putzer, H. \& Onken, R. (2003): COSA. A generic cognitive system architecture based on a cognitive model of human behavior. Cognition, Technology \& Work 5 (2), 140-151.

Schlagowski, R., Merkel, L. \& Meitinger, C. (2017): Design of an assistant system for industrial maintenance tasks and implementation of a prototype using augmented reality. In 2017 IEEE International Conference on Industrial Engineering \& Engineering Management. IEEE IEEM2017 : 10-13 Dec, Singapore (S. 294-298). Piscataway, NJ: IEEE. 
Wang, J., Feng, Y., Zeng, C. \& Li, S. (2014): An augmented reality based system for remote collaborative maintenance instruction of complex products. In IEEE International Conference on Automation Science and Engineering (CASE), 2014. 18 - 22 Aug. 2014, Taipei, Taiwan (S. 309-314). Piscataway, NJ: IEEE.

Wang, X., Ong, S. K. \& Nee, A.Y.C. (2016): Multi-modal augmented-reality assembly guidance based on bare-hand interface. Advanced Engineering Informatics 30 (3), 406-421.

Zhu, J., Ong, S. K. \& Nee, A. Y. C. (2014): A context-aware augmented reality system to assist the maintenance operators. International Journal on Interactive Design and Manufacturing (IJIDeM) 8 (4), 293-304. 